\title{
Compliance With Legal Age Restrictions on Adolescent Alcohol Sales for Alcohol Home Delivery Services (AHDS)
}

\author{
Joris J. Van Hoof \\ University of Twente, Enschede, The Netherlands \\ Esther Van Den Wildenberg and Dorien De Bruijn \\ Dutch Institute for Alcohol Policy - STAP, Utrecht, The Netherlands
}

\begin{abstract}
Purpose: Alcohol availability is an important predictor of alcohol use in adolescents and its negative consequences. Within this study, we zoomed in on availability through alcohol home delivery services (AHDS) by measuring compliance with the legal age limit in this sector. Methods: Two methods were used: (1) mystery shopping in which 21 underage AHDS beer orders were placed and received and (2) mystery calls in which 30 AHDS were called and questioned. Results: In the mystery shopping study, all 21 deliveries were approved and executed by the AHDS (compliance $0 \%$ ). Within the mystery calls, 23 AHDS said they would not sell alcohol to 15 -year-olds ( $76.7 \%$ self-reported compliance), but 19 of them approved secondary purchasing. In total, self-reported compliance was only found in 4 out of the 30 AHDS $(13.3 \%)$. Conclusions: In addition to supermarkets, liquor stores, and the catering industry, AHDS should not be overlooked. Low (or no) compliance with age limits in AHDS is a weak link in the system of underage alcohol availability.
\end{abstract}

Keywords: adolescent alcohol sales, age restrictions, alcohol home delivery services (AHDS), compliance, mystery calls, mystery shopping

\section{INTRODUCTION}

As in many other countries, the sale of alcohol in the Netherlands is restricted to people above a certain age. In order to reduce availability (the strongest predictor of consumption) of alcohol for young people, at the time of this study, customers must be at least 16 years old to buy low $(<15 \%$ alcohol $)$ alcohol containing beverages (beer, wine). However, compliance to this age restriction, as described in the Dutch Licensing and Catering Act, on alcohol sales in supermarkets, liquor stores, and catering industry turns out to be very low $(20 \%$ average) (Gosselt, Van Hoof, Baas, \& De Jong, 2011; Gosselt, Van Hoof, De Jong, \& Prinsen, 2007; Van Hoof, Gosselt, Baas, \& De Jong, 2011).

Address correspondence to Joris J. Van Hoof, PhD, University of Twente, Faculty of Behavioral Sciences, Department of Communication Studies, P.O. Box 217, 7500 AE Enschede, The Netherlands. E-mail: j.j.vanhoof@utwente.nl
In addition to supermarkets, liquor stores, and the catering industry, alcohol home delivery services (AHDS) may be or become a source for underage alcohol sales (Fletcher, Nugent, Ahern, \& Willenbring, 1996; Fletcher, Toomey, Wagenaar, Short, \& Willenbring, 2000). Within AHDS alcohol can be purchased anonymously and comfortably, which is attractive for young customers.

This study was designed to (1) develop a method to measure compliance with the legal age limit of 16 years on alcohol sales by AHDS and (2) give a first indication on AHDS compliance. Both actual compliance and self-reported compliance (by the delivery service) were measured.

\section{METHOD}

To measure compliance, a two-way "mystery shopping" approach was used. First, 15-year-old adolescents ordered (by telephone) and tried to receive (at "home") a crate with 24 bottles (11.16 fluid ounces each) of beer. 
The measurements took place in the six cities within the Netherlands in which at least two AHDS were active. Twelve mystery shoppers (6 15-year-old boys and 6 15-year-old girls) were able to conduct 21 mystery purchase attempts (3 were closed in wave 2). Within each city, two houses were used from which the order was placed and received (not the mystery shoppers' own houses). Second, all home delivery services in the Netherlands were called using "mystery calls" in order to explore if they were willing to sell alcohol to a 15-year-old potential customer. This study was approved by the CEO of the Dutch Institute for Alcohol Policy, and the Ethical Commission of the Faculty of Behavioural Sciences of the University of Twente. All mystery shoppers received some financial compensation for their participation, were educated on alcohol risks, and provided written parental consent.

\section{Selection of Home Delivery Services}

By conducting Internet research, we found 50 beer alcohol home delivery services in the Netherlands (e.g., "Beer Taxi," "911-Beer"). For the mystery home delivery study we selected 17 AHDS, based on geographical location and practical issues (at least 2 AHDS in a city, 2 possible delivery houses, 2 15-year-old mystery shoppers available), of which 12 (wave 1) and 9 (wave 2) could be reached to conduct a mystery home delivery. For the mystery calls we tried to call all 50 AHDS, but only 30 appeared to be in business or reachable.

\section{Protocols}

In the first part of the study, the underage mystery shoppers called 21 AHDS and ordered a crate with 24 bottles of beer, using a pretested "telephone script." In this script all possible questions and answers were depicted (e.g., address, telephone number ["I'll call you when I'm nearby; what's your number?'], nearby streets and shops ['That's close to Fourth Street, right?"]). If the order was accepted (which happened 21 times, out of 21) the address was given and the conversation was ended. The delivery houses were prepared so it seemed that the underage mystery shopper was home alone. When the actual delivery started, the mystery shopper used a "door script," in which she or he lied about her or his age (stated to be 16 years of age), and used her or his legal identification document (which shows they are too young) if asked. Also within this protocol all possible reactions to a deliverer's response were trained.

In the second part of the study, we called all 50 AHDS, of which 30 could be reached by telephone. Using a script (see Figure I), the AHDS were asked if they were willing to make a delivery to an underage

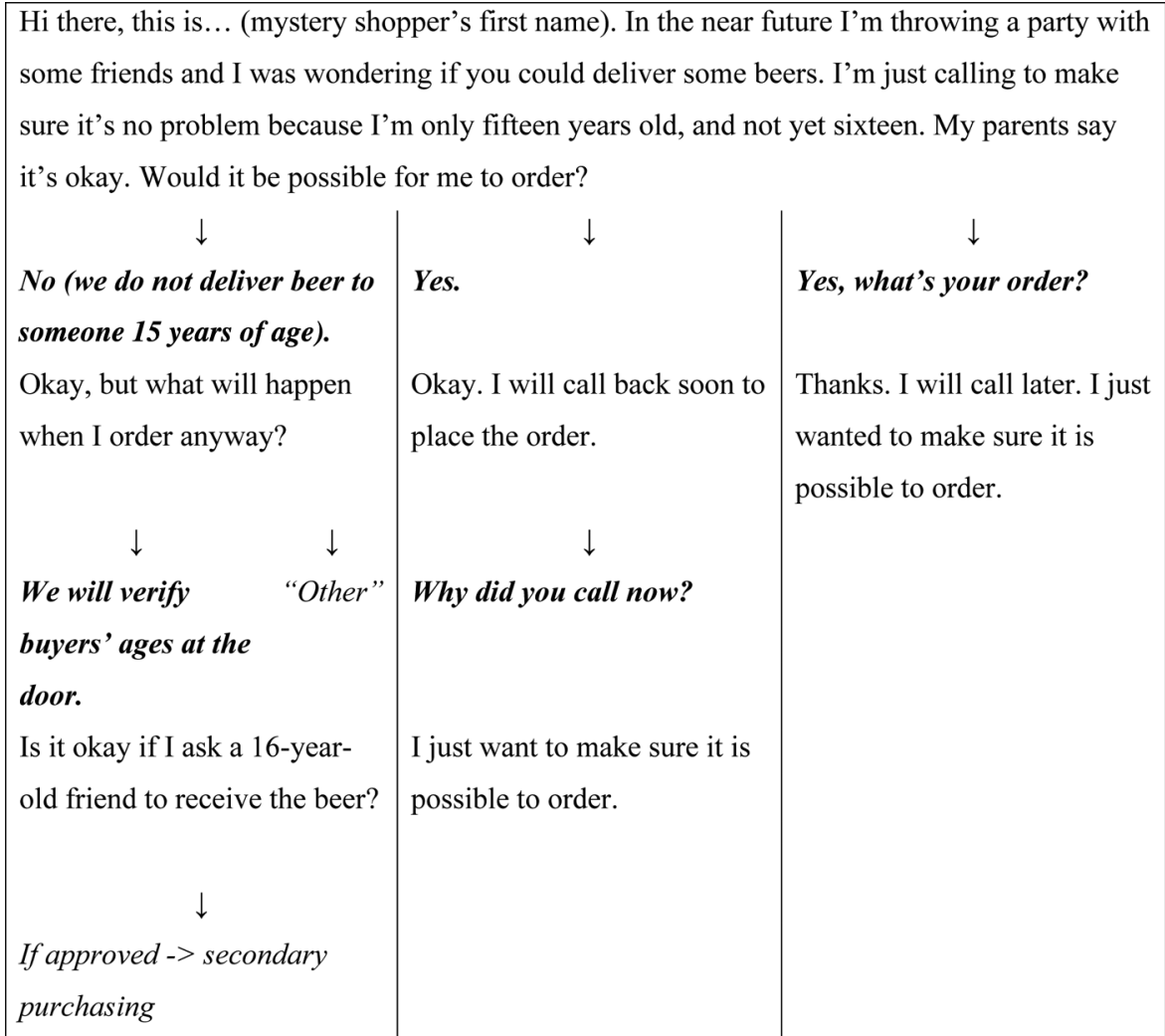

FIGURE 1 Mystery calls script. 
customer. Because approval of secondary purchases is also a legal violation, this was also asked.

\section{RESULTS}

All 21 times, the AHDS turned out to sell alcohol to our underage mystery shoppers, which represents a compliance rate of $0 \%$. Within the 21 deliveries, delivery staff never asked for an identification document (which is obligated in Dutch legislation when a potential customer is not undoubtedly old enough to purchase alcohol).

Within the mystery calls of the 30 AHDS, 7 times $(23.3 \%)$ the direct delivery to a 15 -year-old customer was approved ( 5 times without parental consent and 2 AHDS claimed they needed a parental note); the other 23 AHDS stated they would not deliver to an underage customer. However, 19 of them would approve the order if an older friend would receive the delivery. This is also a violation of Dutch legislation, since the AHDS know this is a secondary purchase. Only 4 out of the 30 AHDS (13.3\%) complied, at least by telephone, with the Dutch Licensing and Catering Act.

\section{DISCUSSION}

The results of this first study on AHDS compliance with the Dutch Licensing and Catering Act show that alcohol sales to underage customers is a major point of concern. The reported compliance in the mystery calls on direct delivery to an underage customer is $76.7 \%$. When we include secondary purchases reported compliance drops to $13.3 \%$, but actual compliance measured in the mystery shopping study turns out to be $0 \%$.

In line with that $0 \%$ compliance is the worrisome AHDS personnel's attitude when it comes to secondary purchases. From the 23 AHDS which state to comply with the legal age limit, 19 AHDS did approve secondary purchasing. This shows that those AHDS employees do not support restrictions on underage drinking. This is worrisome also because some AHDS have the possibility to order online, which is even more easily accessible than ordering over the telephone as we did. It is recommended that the Dutch authorities increase the enforcement capacity on AHDS (e.g., using a mystery shopping approach) in order to increase compliance with age limits.

Since availability in general of risk products (alcohol, tobacco, detrimental media, drugs) is an important predictor of consumption, both in adults and adolescents (Paschall, Grube, \& Kypri, 2009; Pokorny, Jason,
\& Schoeny, 2003), the whole system should be taken into account. In this study we demonstrated the lack of compliance within AHDS when it comes to alcohol. If underage adolescents know that compliance is low in AHDS, and they share that information with friends, this weak link in the system might cause a $100 \%$ alcohol availability for people under the legal age limit (Van Hoof \& Gosselt, 2013). Of course, the same rationale occurs when small tobacco shops continue to sell cigarettes or smokeless tobacco ("dip") to underage customers and supermarkets and bars do not, or when violent games are available through the Internet, if game shops are strict on the sales age limit. The availability system is as strong as the weakest link in the chain.

\section{FUNDING}

This project was supported financially by the Dutch Ministry of Health, Welfare and Sport (Ministerie van Volksgezondheid, Welzijn en Sport).

\section{REFERENCES}

Fletcher, L. A., Nugent, S. M., Ahern, S. M., \& Willenbring, M. L. (1996). The use of alcohol home delivery services by male problem drinkers: A preliminary report. Journal of Substance Abuse, $8,251-261$.

Fletcher, L. A., Toomey, T. L., Wagenaar, A. C., Short, B., \& Willenbring, M. L. (2000). Alcohol home delivery services: A source of alcohol for underage drinkers. Journal of Studies on Alcohol, 61, 81-84.

Gosselt, J. F., Van Hoof, J. J., Baas, N., \& De Jong, M. D. T. (2011). Effects of a national information campaign on compliance with age restrictions for alcohol sales. Journal of Adolescent Health, 49, 97-98.

Gosselt, J. F., Van Hoof, J. J., De Jong, M. D. T., \& Prinsen, S. (2007). Mystery shopping and alcohol sales: Do supermarkets and liquor stores sell alcohol to underage customers? Journal of Adolescent Health, 41, 302-308.

Paschall, M. J., Grube, J. W., \& Kypri, K. (2009). Alcohol control policies and alcohol consumption by youth: A multi-national study. Addiction, 104, 1849-1855.

Pokorny, S. B., Jason, L. A., \& Schoeny, M. E. (2003). The relation of retail tobacco availability to initiation and continued smoking. Journal of Clinical Child \& Adolescent Psychology, 32, 193-204.

Van Hoof, J. J., \& Gosselt, J. F. (2013). Underage alcohol sales-It only takes a minute: A new approach to underage alcohol availability. Journal of Studies on Alcohol and Drugs, 74, 423-427.

Van Hoof, J. J., Gosselt, J. F., Baas, N., \& De Jong, M. D. T. (2011). Improving shop floor compliance with age restrictions for alcohol sales: Effectiveness of a feedback letter intervention. European Journal of Public Health, 22, 737-742. 